\title{
Thermocapillary motion of droplets at large Marangoni numbers
}

\author{
P. Gao *, Z. Yin, W. Hu \\ National Microgravity Laboratory, Institute of Mechanics, Chinese Academy of Sciences, Beijing 100080, PR China
}

Received 29 October 2006; received in revised form 20 April 2007; accepted 18 June 2007

\begin{abstract}
In this paper, the thermocapillary motion problem of drops is investigated using the axisymmetric model. The front-tracking method is employed to capture the drop interface. We find that the migration velocity of the drop is greatly influenced by the temperature field in the drop when Ma is fairly large $(>100)$, which leads to an increase-decrease migration velocity at the beginning of our simulations. (c) 2007 COSPAR. Published by Elsevier Ltd. All rights reserved.
\end{abstract}

Keywords: Thermocapillary migration of drops; Zero-gravity; Front-tracking method

\section{Introduction}

When placed in a surrounding fluid medium where a temperature gradient exists, bubbles or droplets will move from the cold side to migrate to the warm side because differences in interfacial tension can produce a shear stress on both sides of the interface. Such motion is termed thermocapillary migration. This phenomenon is important in the processing of materials in the reduced gravity environment and other applications.

The pioneer work of the migration of bubbles and droplets was carried out by Young et al. (1959) (YGB theory). They gave an analytical expression for the terminal velocity of the drop migration when the Reynolds $(\mathrm{Re})$ and Marangoni (Ma) numbers are sufficiently small compared with unity. Using the matched asymptotic method, Subramanian and Balasubramaniam extended the YGB theory to include the weak non-linear terms and found the analytical solution of thermocapillary migration of bubbles and droplets (Subramanian, 1981; Subramanian, 1983; Balasubramaniam and Subramanian, 1996; Balasubramaniam and Subramanian, 2000).

\footnotetext{
* Corresponding author. Tel./fax: +86 1062615530.

E-mail addresses: gaopeng@imech.ac.cn (P. Gao), zhaohua.yin@ imech.ac.cn (Z. Yin), wrhu@imech.ac.cn (W. Hu).
}

The first two-dimensional simulation on this problem was performed by Szymczyk et al. (1987), who calculated the bubble migration with non-zero Re and large Ma. Balasubramaniam and Lavery (1989) simulated bubble migration with different values of $\mathrm{Re}$ and $\mathrm{Ma}$, and found the scaled bubble velocities only had a modest variation for different $\mathrm{Re}$, but the Marangoni numbers had larger influence on the migration velocity. This work was extended by Ma et al. (1999), and they also approved that in the bubble migration the scaled speed was independent of Ma when Ma was fairly large. There are also some full three-dimensional thermocapillary simulations of deformable viscous drops: Haj-Hariri et al. (1997) simulated an isolated drop motion by using the level-set method to catch the drop interface, and found that the inertial effect on the mobility of viscous drops was weaker than that of gas bubbles; Nas (1995, 2003) adopted the front-tracking method to calculate the thermocapillary interaction of two drops. Similar simulations but with the finite element methods were performed by Wang et al. (2004) to calculate the 3D drop migration.

Before this work, only a few numerical researches have studied the unsteady problem of drop migration for large $\mathrm{Ma}(>100)$, and the migrating phenomenon is still unclear. Traditionally, people would like to focus on the final moving speed of the drop. However, we find that, for large Ma, it takes longer time for the drop to reach the steady velocity and the flow field or temperature pattern before the drop 
reaches the steady state is also more complicated and interesting. In the present research, the drop migration with large $\mathrm{Ma}$ is investigated, and the temperature field, especially the temperature field in the drop, is intensively studied. To precisely capture the drop interface, the front-tracking technique (Unverdi and Tryggvason, 1992; Tryggvason et al., 2001) is employed on the interface. Sections 2 and 3 describe the governing equations and our numerical schemes, respectively. Section 4 presents our numerical results and some discussions.

\section{Governing equations}

In this paper, the spherical drop is surrounded by the continuous phase in a cylindrical container as shown in Fig. 1, and the drop is assumed to keep its spherical shapes through the whole process. Our simulation use the axisymmetric model and the computational domain is $\left\{r, z \mid 0 \leqslant r \leqslant r_{0}, z_{0} \leqslant z \leqslant z_{1}\right\}$. The conservation form of incompressible Navier-Stokes equations in the zero-gravity environment can be written as:

$\frac{\partial u}{\partial r}+\frac{u}{r}+\frac{\partial w}{\partial z}=0$

$\begin{aligned} \frac{\partial(\rho u)}{\partial t}+u \frac{\partial(\rho u)}{\partial r}+w \frac{\partial(\rho u)}{\partial z}= & -\frac{\partial p}{\partial r}+\mu\left[\frac{\partial}{\partial r}\left(\frac{1}{r} \frac{\partial(r u)}{\partial r}\right)+\frac{\partial^{2} u}{\partial z^{2}}\right] \\ & +\frac{\partial \mu}{\partial r}\left(\frac{\partial u}{\partial r}+\frac{\partial u}{\partial r}\right)+\frac{\partial \mu}{\partial z}\left(\frac{\partial w}{\partial r}+\frac{\partial u}{\partial z}\right)\end{aligned}$

$$
\begin{aligned}
\frac{\partial(\rho w)}{\partial t}+u \frac{\partial(\rho w)}{\partial r}+w \frac{\partial(\rho w)}{\partial z}= & -\frac{\partial p}{\partial z}+\mu\left[\frac{1}{r} \frac{\partial}{\partial r}\left(r \frac{\partial w}{\partial r}\right)+\frac{\partial^{2} w}{\partial z^{2}}\right] \\
& +\frac{\partial \mu}{\partial r}\left(\frac{\partial w}{\partial r}+\frac{\partial u}{\partial z}\right)+\frac{\partial \mu}{\partial z}\left(\frac{\partial w}{\partial z}+\frac{\partial w}{\partial z}\right),
\end{aligned}
$$

here the velocity vector is $(u, 0, w), \rho$ and $p$ represent the density and the pressure, respectively, and $\mu$ the dynamic viscosity.

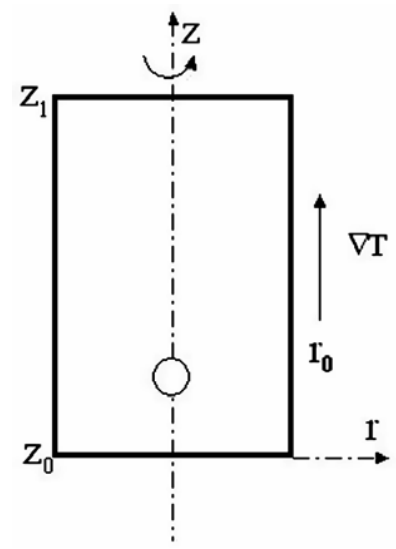

Fig. 1. The schematic diagram of the axisymmetric model for drop Marangoni migration.
The energy equation is

$\frac{\partial(\rho T)}{\partial t}+u \frac{\partial(\rho T)}{\partial r}+w \frac{\partial(\rho T)}{\partial z}=\frac{1}{r} \frac{\partial}{\partial r}\left(r \kappa \rho \frac{\partial T}{\partial r}\right)+\frac{\partial}{\partial z}\left(\kappa \rho \frac{\partial T}{\partial z}\right)$,

where $T$ is temperature and $\kappa$ the thermal diffusivity. The initial conditions of the whole domain are given as

$\left.u\right|_{t=0}=\left.w\right|_{t=0}=0$,

$\left.T\right|_{t=0}=T_{0}+|\nabla T| z$,

where $T_{0}$ is the temperature in the center of the drop at $t=0$ and $\nabla T$ the temperature gradient imposed on the external fluid, which is a constant here. The boundary conditions are (see Fig. 1):

$\left.u\right|_{z=z_{0}}=\left.w\right|_{z=z_{0}}=0,\left.\quad u\right|_{z=z_{1}}=\left.w\right|_{z=z_{1}}=0$,

$\left.u\right|_{r=r_{0}}=\left.w\right|_{r=r_{0}}=0,\left.\quad u\right|_{r=0}=0,\left.\quad \frac{\partial w}{\partial r}\right|_{r=0}=0$,

$\left.T\right|_{z=z_{0}}=T_{0}+|\nabla T| z_{0},\left.\quad T\right|_{z=z_{1}}=T_{0}+|\nabla T| z_{1}$,

$\left.T\right|_{r=r_{0}}=T_{0}+|\nabla T| z,\left.\quad \frac{\partial T}{\partial r}\right|_{r=0}=0$,

The reference velocity $U$ in the fluids is defined as

$U=\left|\sigma_{T}\right||\nabla T| R / \mu_{1}$,

where $R$ is the radius of the drop, and $\left|\sigma_{T}\right|$ the rate of change of interfacial tension with temperature. Throughout this paper, symbols with subscript 1 denotes the physical coefficients of the continuous fluid and those with subscript 2 are the values of the drop. The non-dimensional values $\mathrm{Re}, \mathrm{Ma}$, the ratio of dynamic viscosity and thermal diffusivity are defined as

$\operatorname{Re}=\frac{R U}{v_{1}}, \quad \mathrm{Ma}=\frac{R U}{\kappa_{1}}, \quad \alpha=\mu_{2} / \mu_{1}, \quad \beta=\kappa_{2} / \kappa_{1}$,

Other non-dimensional quantities are:

$\bar{u}=u / U, \quad \bar{r}=r / R, \quad \bar{t}=t /\left(\frac{R}{U}\right), \quad \bar{p}=p /\left(\rho_{1} U^{2}\right)$,

$\bar{T}=T /(|\nabla T| R), \quad \bar{\rho}=\rho / \rho_{1}, \quad \bar{\mu}=\mu / \mu_{1}$,

$\bar{\kappa}=\kappa / \kappa_{1}$,

Thus we get the non-dimensional equations

$$
\begin{aligned}
\frac{\partial \bar{u}}{\partial \bar{r}}+\frac{\bar{u}}{\bar{r}}+\frac{\partial \bar{w}}{\partial \bar{z}}=0, & \\
\frac{\partial(\bar{\rho} \bar{u})}{\partial \bar{t}}+\bar{u} \frac{\partial(\bar{\rho} \bar{u})}{\partial \bar{r}}+\bar{w} \frac{\partial(\bar{\rho} \bar{u})}{\partial \bar{z}}= & -\frac{\partial \bar{p}}{\partial \bar{r}}+\frac{1}{\operatorname{Re}}\left[\bar{\mu}\left(\frac{\partial}{\partial \bar{r}}\left(\frac{1}{\bar{r}} \frac{\partial(\bar{r} \bar{u})}{\partial \bar{r}}\right)+\frac{\partial^{2} \bar{u}}{\partial \bar{z}^{2}}\right)\right. \\
& \left.+\frac{\partial \bar{\mu}}{\partial \bar{r}}\left(\frac{\partial \bar{u}}{\partial \bar{r}}+\frac{\partial \bar{u}}{\partial \bar{r}}\right)+\frac{\partial \bar{\mu}}{\partial \bar{z}}\left(\frac{\partial \bar{w}}{\partial \bar{r}}+\frac{\partial \bar{u}}{\partial \bar{z}}\right)\right],
\end{aligned}
$$




$$
\begin{aligned}
& \frac{\partial(\bar{\rho} \bar{w})}{\partial \bar{t}}+\bar{u} \frac{\partial(\bar{\rho} \bar{w})}{\partial \bar{r}}+\bar{w} \frac{\partial(\bar{\rho} \bar{w})}{\partial \bar{z}} \\
&=-\frac{\partial \bar{p}}{\partial \bar{z}}+\frac{1}{\operatorname{Re}}\left[\bar{\mu}\left(\frac{1}{\bar{r}} \frac{\partial}{\partial \bar{r}}\left(\bar{r} \frac{\partial \bar{w}}{\partial \bar{r}}\right)+\frac{\partial^{2} \bar{w}}{\partial \bar{z}^{2}}\right)\right. \\
&\left.+\frac{\partial \bar{\mu}}{\partial \bar{r}}\left(\frac{\partial \bar{w}}{\partial \bar{r}}+\frac{\partial \bar{u}}{\partial \bar{z}}\right)+\frac{\partial \bar{\mu}}{\partial \bar{z}}\left(\frac{\partial \bar{w}}{\partial \bar{z}}+\frac{\partial \bar{w}}{\partial \bar{z}}\right)\right], \\
& \frac{\partial(\bar{\rho} \bar{T})}{\partial \bar{t}}+\bar{u} \frac{\partial(\bar{\rho} \bar{T})}{\partial \bar{r}}+\bar{w} \frac{\partial(\bar{\rho} \bar{T})}{\partial \bar{z}} \\
&=\frac{1}{\operatorname{Ma}}\left[\frac{1}{\bar{r}} \frac{\partial}{\partial \bar{r}}\left(\bar{r} \bar{\kappa} \bar{\rho} \frac{\partial \bar{T}}{\partial \bar{r}}\right)+\frac{\partial}{\partial \bar{z}}\left(\bar{\kappa} \bar{\rho} \frac{\partial \bar{T}}{\partial \bar{z}}\right)\right],
\end{aligned}
$$

with initial conditions and boundary conditions given as

$$
\begin{aligned}
& \left.\bar{u}\right|_{\bar{t}=0}=\left.\bar{w}\right|_{\bar{t}=0}=0,\left.\quad \bar{T}\right|_{\bar{t}=0}=T_{0} /(|\nabla T| R)+z / R, \\
& \left.\bar{u}\right|_{\bar{z}=z_{0} / R}=\left.\bar{w}\right|_{\bar{z}=z_{0} / R}=0,\left.\quad \bar{u}\right|_{\bar{z}=z_{1} / R}=\left.\bar{w}\right|_{\bar{z}=z_{1} / R}=0, \\
& \left.\bar{u}\right|_{\bar{r}=r_{0} / R}=\left.\bar{w}\right|_{\bar{r}=r_{0} / R}=0,\left.\quad \bar{u}\right|_{\bar{r}=0}=0,\left.\quad \frac{\partial \bar{w}}{\partial \bar{r}}\right|_{\bar{r}=0}=0, \\
& \left.\bar{T}\right|_{\bar{z}=z_{0} / R}=T_{0} /(|\nabla T| R)+z_{0} / R, \\
& \left.\bar{T}\right|_{\bar{z}=z_{1} / R}=T_{0} /(|\nabla T| R)+z_{1} / R, \\
& \left.\bar{T}\right|_{\bar{r}=r_{0} / R}=T_{0} /(|\nabla T| R)+z / R,\left.\quad \frac{\partial \bar{T}}{\partial \bar{r}}\right|_{\bar{r}=0}=0 .
\end{aligned}
$$

In the calculation, $\rho, v$, and $\kappa$ represent functions of physical coefficients which are defined later in Eqs. $(31,32)$.

\section{Numerical methods}

In our computation, we use a fixed, regular, staggered MAC grid and discretize the equations by using a second-order centered differences methods for the spacial variables and the explicit forward Euler scheme in the time integration. The update of the momentum equation is split into two parts. We calculate a prediction velocity $\hat{\bar{u}}, \hat{\bar{w}}$ by employing Eqs. $(2,3)$ and the pressure term is ignored in the first step:

$$
\begin{aligned}
\hat{\bar{u}}= & \bar{u}^{n}-\frac{1}{\bar{\rho}} \Delta \bar{t}\left[\bar{u}^{n} \frac{\partial\left(\bar{\rho} \bar{u}^{n}\right)}{\partial \bar{r}}+\bar{w}^{n} \frac{\partial\left(\bar{\rho} \bar{u}^{n}\right)}{\partial \bar{z}}-\frac{1}{\operatorname{Re}} \bar{\mu}\left(\frac{\partial}{\partial \bar{r}}\left(\frac{1}{\bar{r}} \frac{\partial\left(\bar{r} \bar{u}^{n}\right)}{\partial \bar{r}}\right)+\frac{\partial^{2} \bar{u}^{n}}{\partial \bar{z}^{2}}\right)\right. \\
& \left.-\frac{1}{\operatorname{Re}}\left(\frac{\partial \bar{\mu}}{\partial \bar{r}}\left(\frac{\partial \bar{u}^{n}}{\partial \bar{r}}+\frac{\partial \bar{u}^{n}}{\partial \bar{r}}\right)+\frac{\partial \bar{\mu}}{\partial \bar{z}}\left(\frac{\partial \bar{w}^{n}}{\partial \bar{r}}+\frac{\partial \bar{u}^{n}}{\partial \bar{z}}\right)\right)\right], \\
\hat{\bar{w}}= & \bar{w}^{n}-\frac{1}{\bar{\rho}} \Delta \bar{t}\left[\bar{u}^{n} \frac{\partial\left(\bar{\rho} \bar{w}^{n}\right)}{\partial \bar{r}}+\bar{w}^{n} \frac{\partial\left(\bar{\rho} \bar{w}^{n}\right)}{\partial \bar{z}}-\frac{1}{\operatorname{Re}} \bar{\mu}\left(\frac{1}{\bar{r}} \frac{\partial}{\partial \bar{r}}\left(\bar{r} \frac{\partial \bar{w}^{n}}{\partial \bar{r}}\right)+\frac{\partial^{2} \bar{w}^{n}}{\partial \bar{z}^{2}}\right)\right. \\
& \left.-\frac{1}{\operatorname{Re}}\left(\frac{\partial \bar{\mu}}{\partial \bar{r}}\left(\frac{\partial \bar{w}^{n}}{\partial \bar{r}}+\frac{\partial \bar{u}^{n}}{\partial \bar{z}}\right)+\frac{\partial \bar{\mu}}{\partial \bar{z}}\left(\frac{\partial \bar{w}^{n}}{\partial \bar{z}}+\frac{\partial \bar{w}^{n}}{\partial \bar{z}}\right)\right)\right],
\end{aligned}
$$

where $\Delta \bar{t}$ is the time step. Then, the pressure is determined by solving the equation below to meet the constraint of incompressible condition:

$\frac{1}{\bar{r} \bar{\rho}} \frac{\partial \bar{p}^{n+1}}{\partial \bar{r}}+\frac{\partial}{\partial \bar{r}}\left(\frac{1}{\bar{\rho}} \frac{\partial \bar{p}^{n+1}}{\partial \bar{r}}\right)+\frac{\partial}{\partial \bar{z}}\left(\frac{1}{\bar{\rho}} \frac{\partial \bar{p}^{n+1}}{\partial \bar{z}}\right)=\frac{1}{\Delta \bar{t}}\left(\frac{\partial \hat{\bar{u}}}{\partial \bar{r}}+\frac{\hat{\bar{u}}}{\bar{r}}+\frac{\partial \hat{\bar{w}}}{\partial \bar{z}}\right)$,

The second part is the correction step, where pressure gradient is added

$$
\begin{aligned}
& \bar{u}^{n+1}=\hat{\bar{u}}-\Delta \bar{t} \frac{1}{\bar{\rho}} \frac{\partial \bar{p}^{n+1}}{\partial \bar{r}}, \\
& \bar{w}^{n+1}=\hat{\bar{w}}-\Delta \bar{t} \frac{1}{\bar{\rho}} \frac{\partial \bar{p}^{n+1}}{\partial \bar{z}},
\end{aligned}
$$

The energy equation Eq. (4) is discretized as

$$
\begin{aligned}
\bar{T}^{n+1}= & \bar{T}^{n}-\frac{1}{\bar{\rho}} \Delta \bar{t}\left[\bar{u}^{n+1} \frac{\partial\left(\bar{\rho} \bar{T}^{n}\right)}{\partial \bar{r}}+\bar{w}^{n+1} \frac{\partial\left(\bar{\rho} \bar{T}^{n}\right)}{\partial \bar{z}}\right. \\
& \left.-\frac{1}{M a}\left(\frac{1}{\bar{r}} \frac{\partial}{\partial \bar{r}}\left(\bar{r} \bar{\kappa} \bar{\rho} \frac{\partial \bar{T}^{n}}{\partial \bar{r}}\right)+\frac{\partial}{\partial \bar{z}}\left(\bar{\kappa} \bar{\rho} \frac{\partial \bar{T}^{n}}{\partial \bar{z}}\right)\right)\right],
\end{aligned}
$$

The equations are solved on the fixed grids, but all of the physical coefficients are discontinuous across the interface. It is necessary to convert a quantity which exists on the surface to a fixed grid value. To solve these problems, the front-tracking method [see Unverdi and Tryggvason (1992); Tryggvason et al. (2001) for more details] is employed here. To explicitly mark the position of the interface, an additional computational element - the interface grid is introduced. Meanwhile, an indicator function (defined as a sine function here) is constructed from the known position of the interface to calculate interfacial material properties. For example, the density $\rho$ and kinematic viscosity $v$ are constant within each fluid, and the functions evaluating these variables near the interface are

$$
\begin{aligned}
& \rho(s)=\frac{\rho_{1}-\rho_{2}}{2} \sin \left(\frac{\pi s}{\epsilon}\right)+\frac{\rho_{1}+\rho_{2}}{2}, \\
& v(s)=\frac{v_{1}-v_{2}}{2} \sin \left(\frac{\pi s}{\epsilon}\right)+\frac{v_{1}+v_{2}}{2} .
\end{aligned}
$$

Here, $\epsilon$ is a finite thickness of the transitional zone between the external fluid phase and the drop phase, and $\mathrm{s}(\in[-\epsilon / 2$, $\epsilon / 2])$ the radial displacement from the calculation grid nodes. With these functions the two phases problem can be united to one set of equations and solved on the fixed grids. In our calculation, the surface tension on each calculating element is defined as

$\delta \mathbf{F}_{\sigma}=\int_{\text {element }} \sigma \mathbf{t} \times \mathbf{n d} l$,

where $\mathbf{t}$ is the tangent vector of the edge of the element, and n the normal vector. $\sigma$ is the coefficient of surface tension $\sigma=\sigma_{0}+\sigma_{T}\left(T_{0}-T\right)$,

where $\sigma_{0}$ is the surface tension at a reference temperature $T_{0}$, and $\sigma_{T}$ is a constance depending on the kinds of fluids we used in the simulation.

In our simulation, the surface tension on the interface grid is converted to a body-force-like term using an interpolation function (for details see Peskin (1977)) and transferred to the fixed grid. Similarly, the variables on the fixed grid such as velocity, pressure and temperature can also be transferred onto the interface grid.

To validate our code, we adopt very small $\mathrm{Re}$ and $\mathrm{Ma}$ in our simulation, and the final computed scaled migration velocities are compared with the analytical results of YGB (Fig. 2). The non-dimensional numbers are chosen 


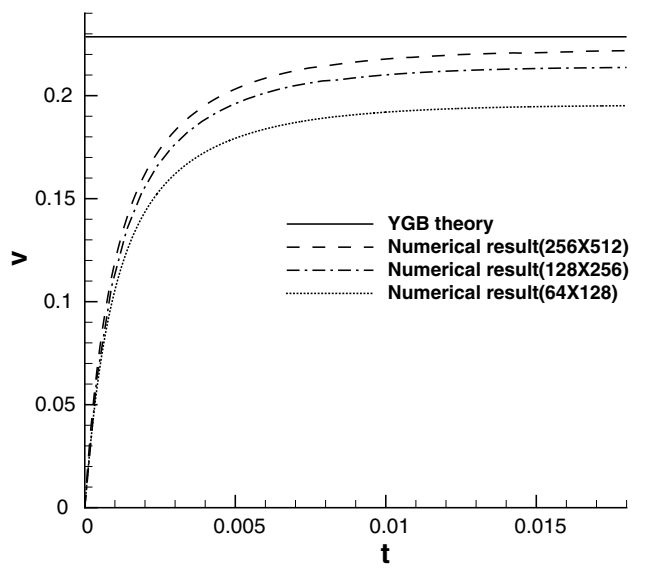

Fig. 2. Time evolutions of drop migration velocity and their comparison with YGB theory. Here, $\mathrm{Re}=\mathrm{Ma}=3 \times 10^{-3}$, with resolutions $64 \times 128$, $128 \times 256$ and $256 \times 512$.

as $\mathrm{Re}=\mathrm{Ma}=3 \times 10^{-3}, \alpha=0.5$, and $\beta=0.5$. A converged trend is found when we increase the resolution, and our result is only $3 \%$ smaller than that of the YGB theory when the grid number is $256 \times 512$.

\section{Results and discussion}

In our simulation, we use Fluorinert liquid FC-75 and 5 cst silicon oil as drop liquid and continuous liquid, respectively. The time step is $0.0005, \alpha=0.3, \beta=0.3$, and the resolution is $128 \times 384$. The computed domain in the calculations is $6 \times 18$ drop radii, and the drop is kept fairly away from the computation boundary through the whole simulation.

At the very beginning of the simulation, the drop center is located at $(r, z)=(0,0)$. The drop, driven by the capillary force, then starts to move towards the warm side along the symmetric $z$-axis. The computed velocity field in the laboratory reference frame, when the migration velocity becomes steady, is shown in Fig. 3a, and the velocity field of reference frame moving with the drop (Fig. 3b), shows that there are two vortices forming in the drop, which agrees well with the YGB theory.

From the definition of $\mathrm{Ma}$, we can see that larger $\mathrm{Ma}$ means the stronger heat convection effect. The temperature re-distribution around the drop interface can change the interface tension of the drop, so it is necessary to analyze the evolution of temperature field. As shown in (Fig. 3), the shear stress on the drop interface will lead the streamline in the drop to form a vortex, which will destroy the initial temperature filed in the drop (Fig. 4a), and the isotherm near the interface will bend along the flow direction. At the same time the temperature fluid in the drop will also be affected by the two rotating vortices in the drop (Fig. $4 \mathrm{~b}-\mathrm{e}$ ). As a result, at $t \approx 8$, the cold part of fluid originally at the bottom of the drop will rise to the top, and the hot fluid on the top will go to the bottom. This temperature switch process will continue until the temperature field becomes stable. At $t \approx 80$ the temperature field is stable as a vortex form, and the coldest part in the drop is the center of the vortex. Notice that the temperature inside the drop will affect the interface tension which drives the drop, leading to the sudden decreased velocity at the very beginning of our simulation.

Fig. 5 is the temperature difference between the top and bottom points on the surface of the drop change along the whole process. The main reason for decrease at the beginning of the curve is the initial movement of the cold temperature fluid at the bottom of the drop, which reduces the temperature at top of the drop. As time goes on, the heat conduction effect around the drop can slowly raise the fluid temperature in the drop. As a result, the temperature difference will be almost stable eventually. When the temperature difference decreases, difference of interface tension will fall too. So the driven force of the drop will be reducing all the time until it is nearly invariable finally.

Time evolutions of velocity are shown in Fig. 6a. Due to the strong heat convection process described above, it

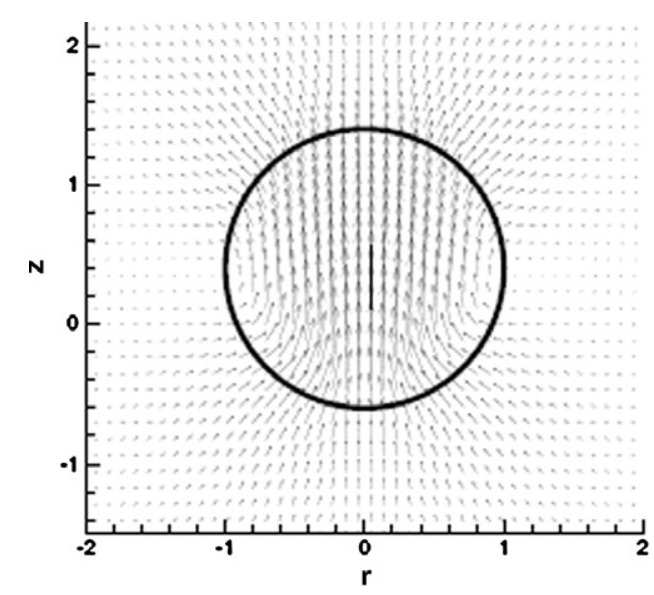

(a) $t=80$

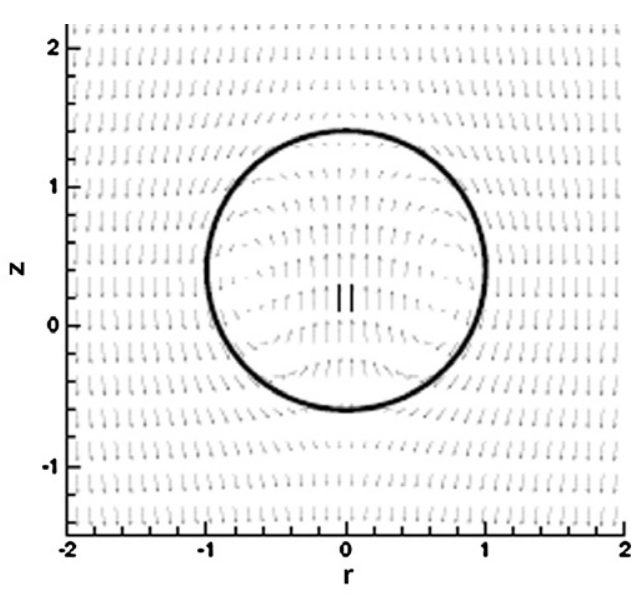

(b) $\mathrm{t}=80$

Fig. 3. The computed velocity field, $\mathrm{Ma}=524.7, \mathrm{Re}=10$. (a) In the laboratory reference frame; (b) In the reference frame moving with the drop. 


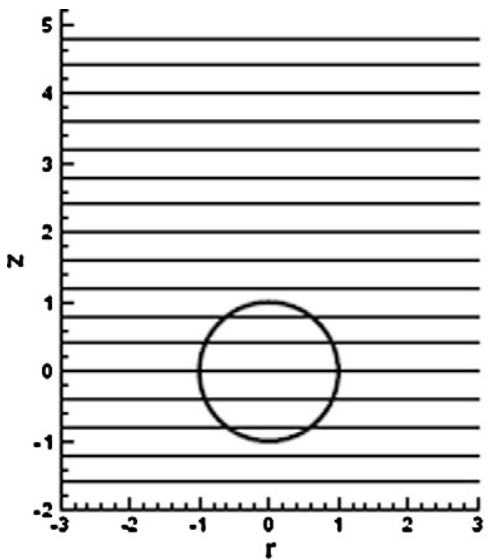

(a) $t=0$

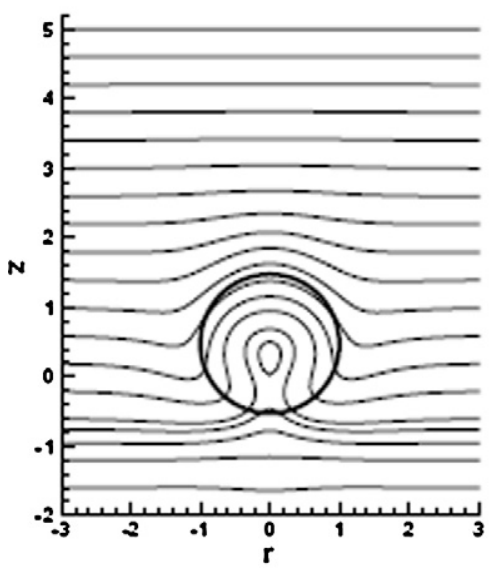

(d) $t=6$

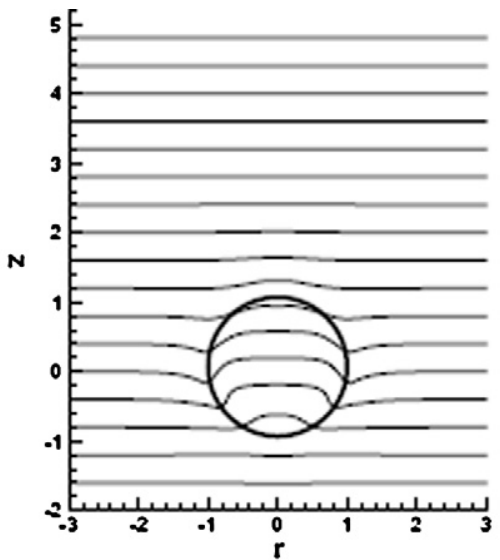

(b) $t=2$

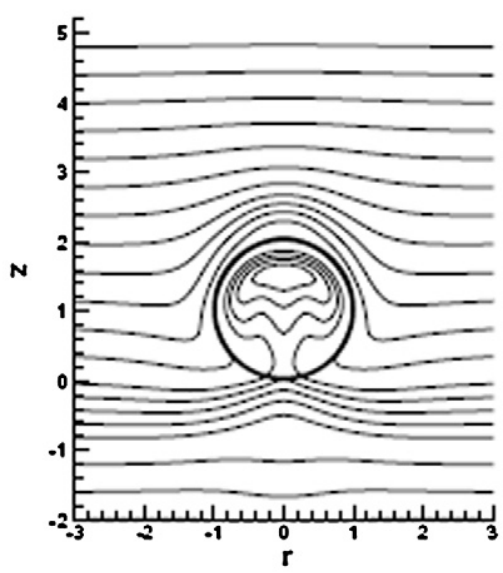

(e) $t=12$

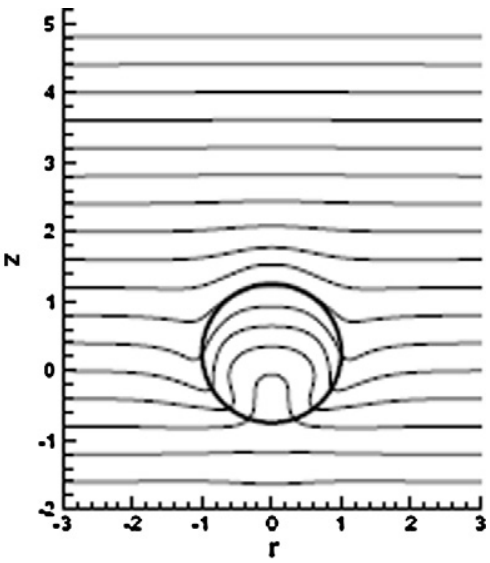

(c) $t=4$

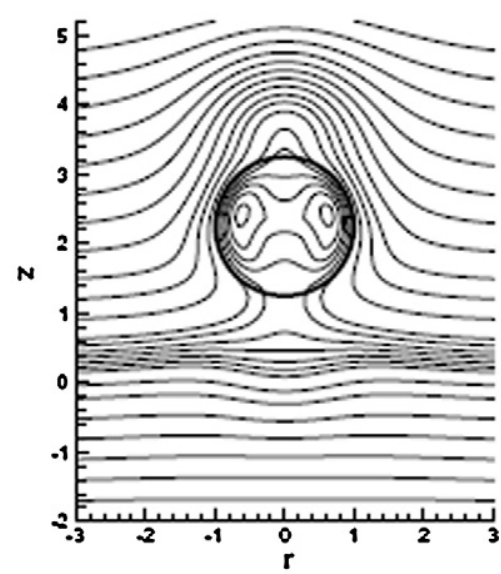

(f) $t=90$

Fig. 4. The evolution of temperature field in the drop migration. $\mathrm{Ma}=524.7, \mathrm{Re}=10$.

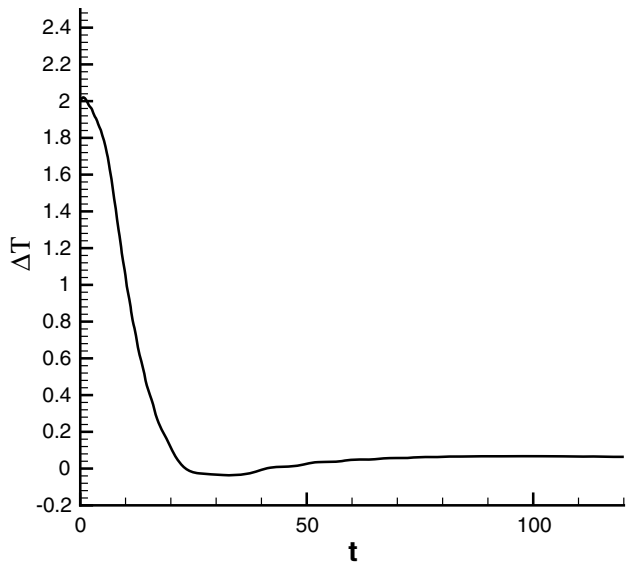

Fig. 5. Temperature difference between the forward and rear stagnation points on the surface of the drop. $\mathrm{Ma}=524.7, \mathrm{Re}=10$.

appears that after a short period of increase at the very beginning of the simulation, the migration velocity starts to drop until it reaches a steady state. This phenomenon was reported in previous work but without any detailed analysis. We can find the similar trend of the drop migration velocity curve in Nas's work (Nas and Tryggvason, 2003), where Ma was below 100, however they did not give the explanation of this. In our paper, the $\mathrm{Ma}$ is much larger, and the decreasing of the drop migration velocity is more apparent.

It should be noticed that large Ma means the effect of heat convection is much larger than that of heat diffusion. For small Ma, the heat convection process inside the drop will still transport the cold fluids up to the top of the drop, but it happens so slowly that the cold liquid has already been warmed up before it reaches the top. Thus, when the $\mathrm{Ma}$ is small, the heat convection effect can hardly change the temperature difference on the drop interface. With the same Re but different Ma, Fig. 6b shows that, when $\mathrm{Ma}$ is small enough, the increase-decrease process at the beginning of the simulation disappears. As a result, the time to reach the equilibrium state in the case of $\mathrm{Ma}=1$ is only $40 \%$ of that of large Ma. More details about this small-Ma simulation will not be shown here to keep this paper concise. 

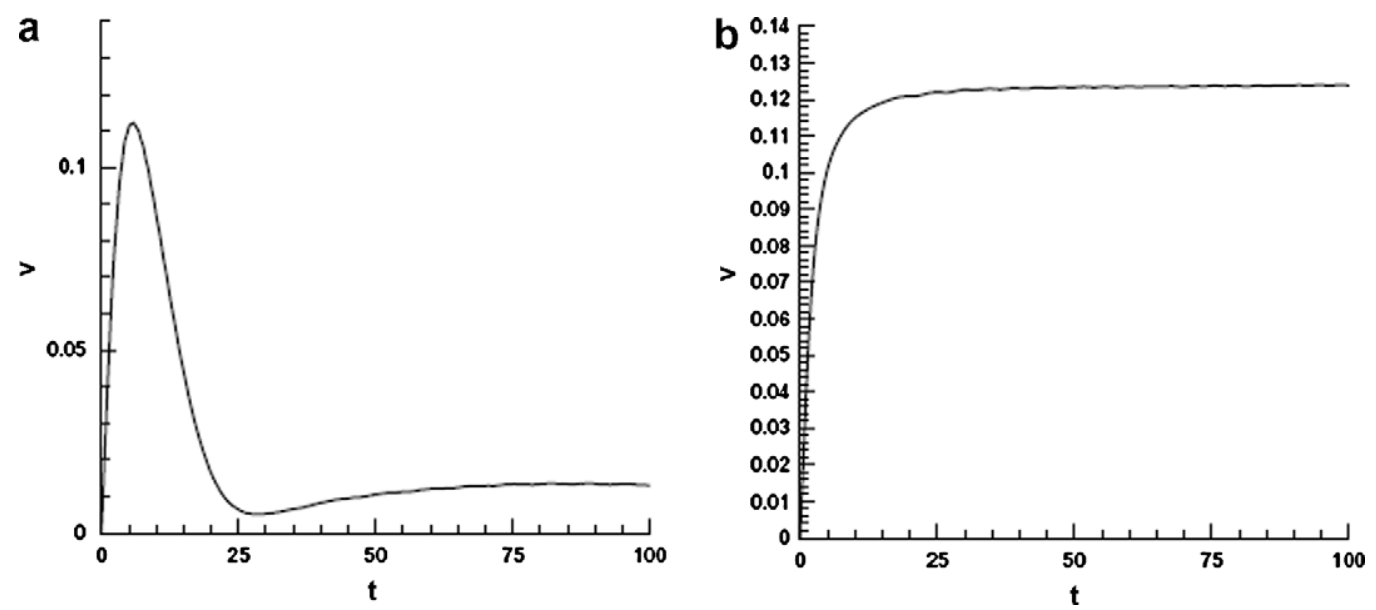

Fig. 6. The migration velocity of the drop is plotted versus time. (a) $\mathrm{Ma}=524.7, \mathrm{Re}=10$; (b) $\mathrm{Ma}=1, \mathrm{Re}=10$. Both the velocity and the time are nondimensional, and the non-dimensional velocity is scaled by $\mathrm{U}$ defined in Eq. (11).

\section{Conclusions}

In this paper, we have numerically investigated the thermocapillary migration of spherical drops in continuous phase fluid under imposed temperature gradient. Fronttracking method is used here to solve this unsteady problem. We find that the migration velocity can not reach a steady value directly when the Ma is large $(>100)$. We also give the reason for this overshoot phenomenon through analyzing the evolution of temperature field during whole migration process. In the future, we will consider the deformation effect of the drop and give more detailed discussions for different sets of $\mathrm{Re}$ and Ma.

\section{Acknowledgements}

We thank Prof. Xi-Yun Lu and Prof. Ze-Mei Tang for useful discussions. This project is supported by NSF of China (G10502054 and G10432060).

\section{References}

Young, N.O., Goldstein, J.S., Block, M.J. The motion of bubbles in a vertical temperature gradient. J. Fluid Mech. 6, 350-356, 1959.

Subramanian, R.S. Slow migration of a gas bubble in a thermal gradient. A.I.Ch.E. J. 27 (4), 646-654, 1981.

Subramanian, R.S. Thermocapillary migration of bubbles and droplets. Adv. Space Res. 3 (5), 145-153, 1983
Balasubramaniam, R., Subramanian, R.S. Thermocapillary bubble migration - thermal boundary layers for large Marangoni numbers. Int. J. Multiphase Flow 22 (3), 593-612, 1996.

Balasubramaniam, R., Subramanian, R.S. The migration of a drop in a uniform temperature gradient at large Marangoni numbers. Phys. Fluids 12 (4), 733-743, 2000.

Szymczyk, J.A., Wozniak, G., Siekmann, J. On Marangoni bubble motion at higher Reynolds- and Marangoni-numbers under microgravity. Appl. Microgravity Tech. 1 (1), 27-29, 1987.

Balasubramaniam, R., Lavery, J.E. Numerical simulation of thermocapillary bubble migration under microgravity for large Reynolds and Marangoni numbers. Num. Heat Transfer A 16, 175-187, 1989.

Ma, X.J., Balasubramaniam, R., Subramanian, R.S. Numercial simulatoin of thermocapillary drop motion with internal circulation. Num. Heat Transfer A 35, 291-309, 1999.

Haj-Hariri, H., Shi, Q., Borhan, A. Thermocapillary motion of deformable drops at finite Reynolds and Marangoni numbers. Phys. Fluids 9 (4), 845-855, 1997.

S. Nas. Computational investigation of thermocapillary migration of bubbles and drops in zero gravity. Ph.D. Dissertation, University of Michigan (1995).

Nas, S., Tryggvason, G. Thermocapillary interaction of two bubbles and drops. Int. J. Multiphase Flow 29, 1117-1135, 2003.

Wang, Y.X., Lu, X.Y., Zhuang, L.X., Tang, Z.M., Hu, W.R. Numerical simulation of drop Marangoni migration under microgravity. Acta. Astronautica 54, 325-335, 2004.

Unverdi, S.O., Tryggvason, G. A front-tracking method for viscous incompressible flows. J. Comput. Phys. 100, 25-37, 1992.

Tryggvason, G., Bunner, B., Esmaeeli, A., Juric, D., Al-Rawahi, N., Tauber, W., Han, J., Nas, S., Jan, Y.-J. A front-tracking method for computations of multiphase flow. J. Comput. Phys. 196, 708-759, 2001.

Peskin, C.S. Numerical analysis of blood flow in the heart. J. Comput. Phys. 25, 220-225, 1977 